\title{
The effect of photo-generated carriers on the spectral diffusion of a quantum dot coupled to a photonic crystal cavity
}

\author{
Arka Majumdar,* Erik D. Kim, and Jelena Vučković \\ ${ }^{1}$ E.L.Ginzton Laboratory, \\ Stanford University, Stanford, CA, 94305
}

\begin{abstract}
We experimentally observe the effect of photo-generated carriers on the spectral diffusion of a quantum dot (QD) coupled to a photonic crystal (PC) cavity. In this system, spectral diffusion arises in part from charge fluctuations on the etched surfaces of the PC. We find that these fluctuations may be suppressed by photo-generated carriers, leading to a reduction of the measured QD linewidth by a factor of $\sim 2$ compared to the case where the photo-generated carriers are not present. This result demonstrates a possible means of countering the effects of spectral diffusion in QD-PC cavity systems and thus may be useful for quantum information applications where narrow QD linewidths are desired.
\end{abstract}

\section{INTRODUCTION}

A single self-assembled quantum dot (QD) coupled to a high-quality (Q) factor semiconductor optical cavity has emerged in recent years as a promising system for developing on-chip quantum technologies [1-3]. Although this solid-state cavity quantum electrodynamic (CQED) system behaves similarly to its atomic counterpart in a number of ways, the unavoidable interaction of the QD with its solid-state environment gives rise to phenomena specific to the solid-state system. For instance, the presence of acoustic phonons even at cryogenic temperatures has led to the observation of far off-resonant coupling between a QD and a cavity, where the resonant excitation results in the emission of a photon at the cavity (QD) wavelength when the QD (cavity) is driven [4-6]. In this case, the phonon-mediated off-resonant coupling may provide a useful means of performing quantum state readout [7].

Another important phenomenon arising from the solid state nature of the QD-cavity system is the random trapping and untrapping of charges in the vicinity of the QD due to defects, leading to spectral diffusion of the QD optical transition energy [8-11]. Spectral diffusion (or jitter) is generally considered detrimental to the optical properties of QDs as it leads to the broadening of optical emission lines [12-14]. Studies of spectral diffusion in bulk self-assembled QD systems have attributed its observation to defects provided by the highly disordered QD wetting layer $[9,15]$. Additionally, it has been postulated that the proximity of etched surfaces near the QD can lead to spectral diffusion due to defects provided by the etched surface roughness [16]. Recent experimental studies of the linewidth of a QD off-resonantly coupled to a photonic crystal (PC) cavity mode have also observed broadening of QD linewidths that cannot simply be attributed to a pure dephasing process [17]. This is

*Electronic address: arkam@stanford.edu not surprising considering that in QD-PC cavity systems both the wetting layer and the presence of etched surfaces are expected to contribute to spectral diffusion, particularly for QDs close to the holes of the photonic lattice. Further, each contribution is expected to manifest under different system conditions. For instance, wetting layer defects predominantly trap charges through the ionization of excitons generated in the vicinity of the QD by above-band (AB) optical excitation. This contribution to spectral diffusion has been shown to depend on the strength of the $\mathrm{AB}$ laser and is absent at very low excitation powers [9]. For trap states provided by the rough etched surfaces of the photonic crystal, fluctuating surface charges are anticipated to contribute to spectral diffusion even in the absence of $\mathrm{AB}$ optical excitation. It is unclear, however, how this contribution to spectral diffusion may be affected by above-band excitation, if it can be affected at all.

Here, we experimentally analyze the dependence of spectral diffusion on the power of an above-band laser for a QD off-resonantly coupled to a PC cavity. Specifically, we perform QD linewidth measurements by scanning a narrow-bandwidth continuous-wave (CW) laser across the QD resonance and observing optical emission from the detuned cavity. In the absence of above-band pumping, we observe a broadened QD linewidth and attribute the broadening to fluctuating surface charges on the nearby etched surfaces. Surprisingly, we find that this contribution to spectral diffusion is suppressed in the presence of above-band excitation. We attribute this suppression to the filling of trap states in nearby etched surfaces by photo-generated carriers, thereby drastically reducing charge fluctuations. This in turn dramatically reduces the experimentally observed QD linewidth. For low $\mathrm{AB}$ laser powers, spectral diffusion becomes negligible and the QD linewidth exhibits the standard power broadening as the $\mathrm{CW}$ laser power is increased. These results demonstrate an optical excitation regime in which spectral diffusion may be overcome and provide a simple means of countering the degradation of the optical properties of QDs in PC structures. This approach could thus 
prove useful in QD-PC cavity based quantum technologies that benefit from narrower emitter linewidths.

\section{QD LINEWIDTH BROADENING}

The effect of the spectral diffusion is reflected in the measurement of the QD linewidth. In the linear response regime, the QD lineshape is characterized by the QD susceptibility [18]

$$
\chi(t) \propto \mathcal{F}\left[e^{\left(i \omega_{0}-\frac{\gamma\left(\omega_{0}\right)}{2}\right) t}\left\langle e^{-i \int_{0}^{t} d \tau \delta \omega(\tau)}\right\rangle\right]
$$

where $\mathcal{F}$ denotes the Fourier transform, $\omega_{0}$ is the QD resonance frequency, $\gamma\left(\omega_{0}\right)$ is the radiative QD linewidth and the statistical average is taken over all the possible frequencies at different times $\tau$. The effect of spectral diffusion is reflected in this statistical average. This average depends on the underlying probability distribution of the random environment. Assuming a Gaussian fluctuation, by Cumulant expansion (and noting the odd moments are zero), we can write

$$
\left\langle e^{-i \int_{0}^{t} d \tau \delta \omega(\tau)}\right\rangle=e^{-\frac{1}{2} \int_{0}^{t} d \tau_{1} \int_{0}^{t} d \tau_{2}\left\langle\delta \omega\left(\tau_{1}\right) \delta \omega\left(\tau_{2}\right)\right\rangle}
$$

Assuming an exponential correlation function (with variance $\Gamma$ and correlation time $\tau_{c}$ ) of the form

$$
\left\langle\delta \omega\left(\tau_{1}\right) \delta \omega \tau_{2}\right\rangle=\Gamma^{2} e^{-\frac{\left|\tau_{1}-\tau_{2}\right|}{\tau_{c}}}
$$

we find that

$$
\left\langle e^{-i \int_{0}^{t} d \tau \delta \omega(\tau)}\right\rangle=e^{-\Phi(t)}
$$

with

$$
\Phi(t)=\Gamma^{2} \tau_{c}^{2}\left[e^{-t / \tau_{c}}+t / \tau_{c}-1\right]
$$

Hence, we can model the effect of the fluctuating environment by two quantities: the standard deviation $\Gamma$ and the correlation time $\tau_{c}$. In the limit of long measurement time $\left(t>>\tau_{c}\right)$, the linewidth of the QD is given by $\left(\gamma+\Gamma^{2} \tau_{c}\right)$, where $\gamma$ comes from the Lorentzian lineshape of the QD and $\Gamma^{2} \tau_{c}$ is the contribution of the fluctuating environment. In this article, we perform two separate sets of experiments to determine these characteristic quantities of the fluctuating environment. From the first set of experiments, we obtain a measure of the lifetime of the charges in the traps and estimate the correlation time $\tau_{c}$ to be approximately $100 \mathrm{~ns}$. In the second set of experiments, we show how photo-generated carriers can mitigate the effect of the spectral diffusion that is present in the absence of any above-band excitation. From experimental results (see section IV) we determine the broadening due to the fluctuating environment and estimate the quantity $\Gamma^{2} \tau_{c}$ to be approximately $8.7 \mathrm{GHz}$, corresponding to $\Gamma=300 \mathrm{MHz}$. We note that as these values were obtained for two different QDs on the same wafer, they provide only order-of-magnitude estimates.

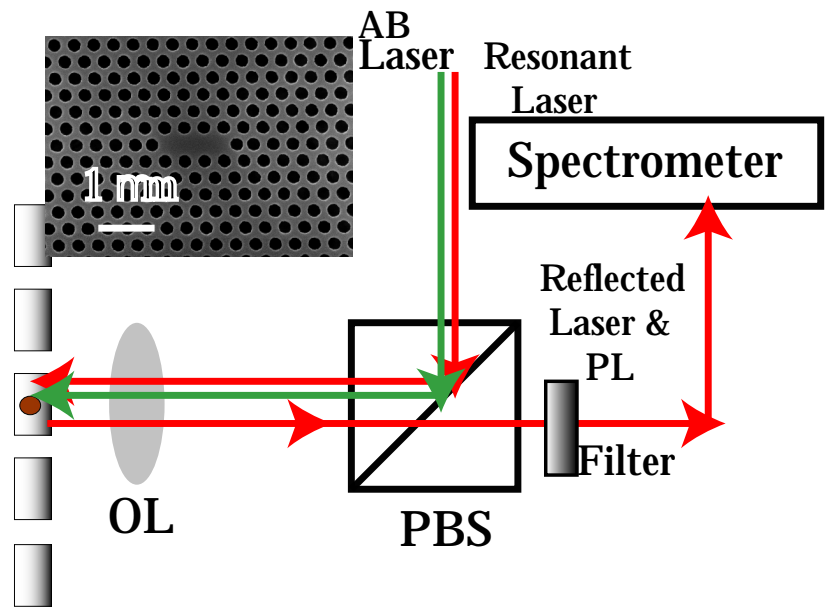

FIG. 1: (color online) The cross-polarized confocal microscopy setup used to probe the coupled QD-cavity system. The inset shows a scanning electron micrograph of the fabricated PC cavity.

\section{QD CHARGING AND MODULATION}

All the experiments are performed in a helium-flow cryostat at cryogenic temperatures $(\sim 30-55 \mathrm{~K})$ on self-assembled InAs QDs embedded in a GaAs photonic crystal cavity $[2,4]$. The $160 \mathrm{~nm}$ GaAs membrane used to fabricate the photonic crystal is grown by molecular beam epitaxy on top of a GaAs (100) wafer. The GaAs membrane sits on a $918 \mathrm{~nm}$ thick sacrificial layer of $\mathrm{Al}_{0.8} \mathrm{Ga}_{0.2} \mathrm{As}$. Under the sacrificial layer, a 10-period distributed Bragg reflector, consisting of a quarter-wave AlAs/GaAs stack, is used to increase the collection into the objective lens [2]. The photonic crystal was fabricated using electron beam lithography, dry plasma etching, and wet etching of the sacrificial layer in hydrofluoric acid $(6 \%)$. The inset of Fig. 1 shows the scanning electron micrograph of a fabricated photonic crystal cavity. A cross-polarized confocal microscopy setup is used to probe the QDs, both in photoluminescence (PL) as well as under resonant excitation as shown in Fig. $1[2,4]$.

In the first set of experiments, we investigate the time scale on which carriers generated by the above-band (AB) laser affect the QD environment. These experiments are used to provide a measure of correlation time $\tau_{c}$ associated with the fluctuating surface charges. To perform these measurements, we first identify a strongly coupled QD-cavity system in PL studies by observing the anticrossing of the QD and cavity resonances that occurs as the temperature is varied. We then probe the system by measuring the transmission of a resonant continuous wave laser through the cavity. In the absence of the $A B$ laser excitation, we observe a Lorentzian line shape for the cavity transmission, indicating that there is no QD coupled to the cavity despite the fact that coupling is observed in PL studies. When the weak AB laser is turned on, however, we observe a drastic change in the transmis- 
sion spectrum as shown in Fig. 2a. The spectrum now appears to show two QDs strongly coupled to the cavity as indicated by the presence of two dips in the transmission spectrum. This dramatic change can be caused either due to capturing of optically generated carriers by the QD [19], or due to decreased spectral diffusion, and thus reduction of the QD linewidth, as will be discussed later in the paper. We note that in presence of the $\mathrm{AB}$ laser, the amount of light coming out of the cavity due to PL is negligible compared to the light transmitted through the cavity.

Next, we study the time dynamics of this resonance shift to obtain a measure of the time scale on which the charge environment surrounding the QD fluctuates. This is done by measuring the transient response of the transmitted resonant laser power to modulation of the $\mathrm{AB}$ laser. We note that this type of modulation is different from the all-optical modulation reported in [20], where the QD shifts continuously with increasing $\mathrm{AB}$ laser power and the modulation is caused by the screening of the built-in electric field by photo-generated carriers. To obtain optimal modulation contrast we tune the CW laser to the dip in the cavity transmission spectrum (shown by the arrow in Figure $2 \mathrm{a}$ ). Figure $2 \mathrm{~b}$ shows the DC modulation behavior when the above-band laser is manually turned on and off at varying time intervals and the resonant laser transmission is measured at steady-state. To measure temporal dynamics, we apply a square-wave modulation to the above-band laser and measure the CW laser transmission by a single photon counter and pico-second time analyzer triggered by the modulated above-band laser [21]. We note that for this experiment, the power of the above-band laser and the resonant CW laser are, respectively, $15 \mathrm{nW}$ and $250 \mathrm{nW}$ as measured in front of the objective lens of the confocal microscopy setup. Figure $2 \mathrm{c}$ shows the modulated cavity transmission output with time, for a modulation frequency of $2 \mathrm{MHz}$. We observe an exponential decay of the resonant laser transmission when the $\mathrm{AB}$ laser is turned off. This is due to the fact that charge carriers remain trapped for some amount of time even after the $\mathrm{AB}$ laser is turned off. We fit the exponential decay and obtain a decay time constant of $\sim 100 \mathrm{~ns}$, corresponding to a maximum modulation speed of $\sim 10 \mathrm{MHz}$. Finally Fig. $2 \mathrm{~d}$ shows the on-off-ratio of the transmitted CW laser as a function of the modulation frequency of the above-band laser, which shows that the maximum modulation speed is approximately on the order of $\sim 10 \mathrm{MHz}$. From this experiment we estimate the time-scale of the fluctuation of charges to be $\sim 100 \mathrm{~ns}$.

\section{QD LINEWIDTH BROADENING}

Having obtained a measure of the correlation time associated with local charge fluctuations, we now turn to experimental measurements of the broadening of QD linewidths by spectral diffusion. For this set of exper-

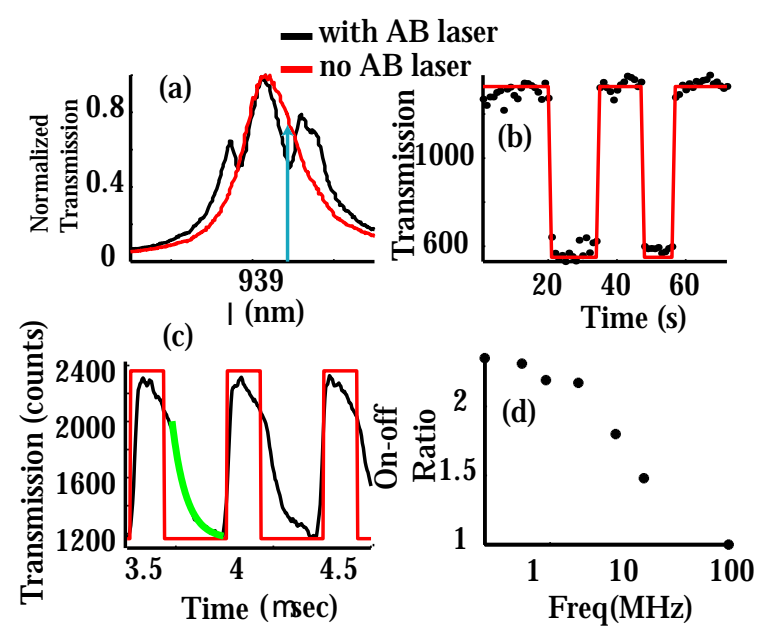

FIG. 2: (color online) (a) Effect of the above-band (AB) laser on the transmission spectrum of a strongly coupled QD-cavity system. The arrow shows the wavelength of the other (resonant) CW laser, whose transmission is modulated by the $\mathrm{AB}$ laser in (b) and (c). (b) The transmitted laser power in steady state when the AB laser is turned on and off manually, at intervals of several 10s. This shows the DC characteristic of the modulation. (c) The temporal dynamics of the QDcavity system: the transmitted laser output as a function of time (black plot). The shown modulation signal (red) is used to modulate the above-band laser. From an exponential fit (green) we estimate a time constant of $\sim 100 \mathrm{~ns}$. (d) The on-off ratio of the transmitted laser as a function of the modulation frequency.

iments, we consider a system where a QD is far offresonantly coupled to a PC cavity mode. For this particular system the dot is $1.4 \mathrm{~nm}$ blue-detuned from the cavity (the dot and cavity are, respectively, at 934.9 and $936.3 \mathrm{~nm}$; see Figure $3 \mathrm{a}$ ). QD linewidths are obtained by measuring the off-resonant cavity emission as the $\mathrm{CW}$ laser is scanned through the QD resonance, similar to experiments reported in Ref. [17]. We observe saturation of the cavity emission and power broadening of the QD with increasing excitation laser power. We emphasize that in the experimental data shown in Fig. 3, the additional above-band excitation is not used, and the QD is driven only by the resonant laser. By simultaneously fitting the cavity emission with the model $I_{\text {sat }} \tilde{P} /(1+\tilde{\tilde{P}})$ (Figure 3 b) and the QD linewidth with the model $\Delta \omega_{0} \sqrt{1+\tilde{P}}$ (Figure $3 \mathrm{c}$ ) [22], we find that the observed QD linewidth is significantly smaller than what is expected from ordinary power broadening of a two level system (the dashed line in Figure $3 \mathrm{c}$ ). Here, $I_{\text {sat }}$ is the saturated cavity emission intensity, $\Delta \omega_{0}$ is the QD linewidth at the limit of zero excitation power and $\tilde{P}$ is the proportional to the laser intensity at the position of the QD. Simple theoretical analysis shows that the QD linewidth exhibits power broadening similar to that of a two-level system [23], thus showing that the cavity read-out does not affect the QD linewidth significantly. This unusual broadening behav- 
ior cannot be explained by an increasing pure dephasing rate with increasing laser power or excitation induced dephasing [24], since those effects would lead to a broader linewidth than that predicted by standard power broadening. In fact, the nature of the broadening indicates a power-independent broadening mechanism.

We thus consider spectral diffusion as a means of modeling this additional power-independent broadening. We note that spectral diffusion is indeed power independent when the laser is resonant with the QD. The effect of spectral diffusion is modeled as a Voigt lineshape, which is a convolution of a Lorentzian lineshape (the actual QD linewidth $\Delta \omega_{L}$ ) and a Gaussian lineshape (the spectral fluctuations with a linewidth of $\left.\Delta \omega_{D}\right)$. The full width half maximum $\Delta \omega_{V}$ of a Voigt line-shape is given by [25]:

$$
\Delta \omega_{V} \approx A \Delta \omega_{L}+\sqrt{(1-A)^{2} \Delta \omega_{L}^{2}+\Delta \omega_{D}^{2}}
$$

where the parameter $A=0.5346$ is empirically determined. We use this model to fit the power broadened linewidth, where $\Delta \omega_{L}=\Delta \omega_{0} \sqrt{1+\tilde{P}}$. The model fits the experimental data very well, with parameters $\Delta \omega_{0} / 2 \pi=4.7 \mathrm{GHz}$ and $\Delta \omega_{D} / 2 \pi=8.7 \mathrm{GHz}$. These results support the theory that spectral diffusion is caused by the empty traps present on the etched surfaces of the photonic crystal. These traps can be randomly charged and discharged, resulting in a fluctuating charge environment that can modify the $\mathrm{QD}$ resonance frequency by random DC Stark shifts. As we perform time-averaged measurements on time scales much longer than the correlation time estimated in the preceding set of experiments, the measured QD linewidth is a statistical mixture of the QD resonances at different resonance frequencies.

We now repeat these experiments in the presence of an above-band laser. In this system, although the QD transition energy is not shifted in the presence of the PL laser, we observe a significant reduction in the measured QD linewidth (by a factor of $\sim 2$ ) and an increase in the off-resonant cavity emission (Figure 4). The resonant laser power is kept at the same value $(\sim 100 \mathrm{nW})$ as used in the measurements of Fig. 3. The increased background in the cavity emission is caused by the PL generated by the $\mathrm{AB}$ laser. We find that the QD exhibits a Lorentzian line-shape in presence of the $\mathrm{AB}$ laser, but shows a nearly Gaussian lineshape in the absence of the $\mathrm{AB}$ laser. We attribute this difference to the fact that one of the effects of the $\mathrm{AB}$ laser is to create carriers that subsequently fill all the empty traps near the QD, thereby reducing spectral fluctuations. The dependence of the QD linewidth as a function of above-band laser power is shown in the inset of Fig. 4. With increasing AB laser power, the PL adds noise to the off-resonant cavity emission, making linewidth measurements difficult. However, we do observe a slight increase in the QD linewidth with increasing $\mathrm{AB}$ laser power, consistent with previous studies [9]. We note that an AB laser can cause multiple effects such as an abrupt change in the QD resonance
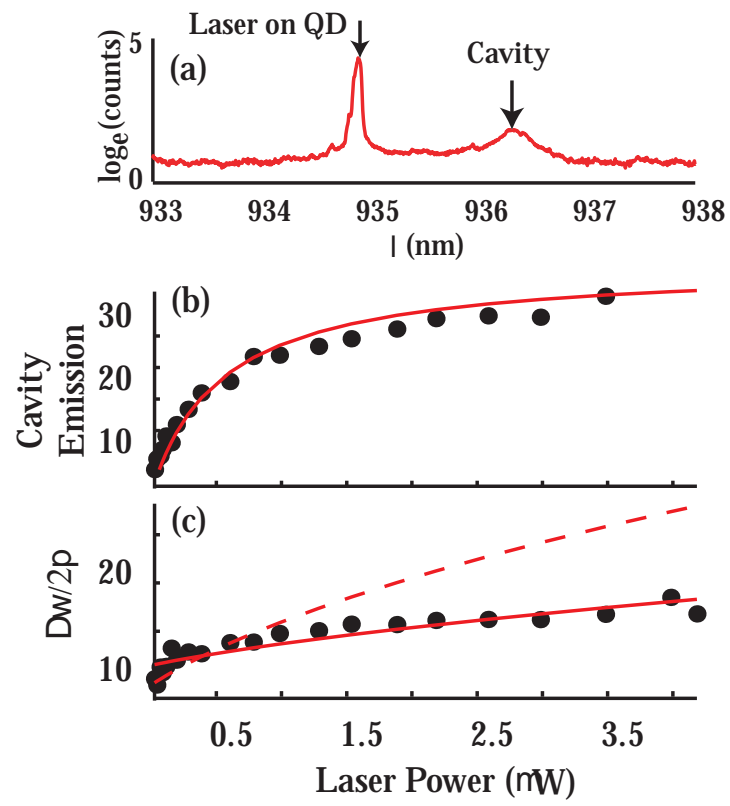

FIG. 3: (color online) QD spectroscopy by off-resonant dotcavity coupling: (a) The spectrum showing the laser resonantly driving the QD and the emission from an off-resonant cavity. A logarithmic scale is used to show both the strong laser and the weak cavity emission. (b) Cavity emission as a function of the power of the laser that is driving the QD resonantly. (c) QD linewidth $\Delta \omega$ measured by monitoring the cavity emission by scanning the laser across the QD. $\Delta \omega$ is plotted as a function of the excitation laser power. We find that the standard power broadening model over-estimates the measured QD linewidth (the dashed line). However, the model provides better agreement with experimental results when spectral diffusion is taken into account (solid line).

frequency [19], or saturation of the QD. Hence this type of linewidth narrowing in the presence of the $\mathrm{AB}$ laser is not observed for all the QDs. However, the additional power-independent broadening is present in the absence of above-band pumping for all QDs studied.

For this particular QD, we perform a power dependent study of the QD linewidth and cavity emission in the presence of the $\mathrm{AB}$ laser. The $\mathrm{AB}$ laser power is kept constant throughout experiments. The data in the absence of the AB laser is the same as shown in Figure 3. Figure 5 a shows the off-resonant cavity emission intensity as a function of the excitation laser power both in the presence and absence of the $\mathrm{AB}$ laser. In the presence of the AB laser, we observe an increased cavity emission and also a saturation trend at lower laser power (shown by the fit). However, with increasing excitation laser power, the cavity emission decreases. This decrease in the cavity emission may be due to the presence of other QD excitonic states that the resonantly pumped QD is coupled to. The fact that those states are present only with the $\mathrm{AB}$ excitation may be an indication that these other ex- 


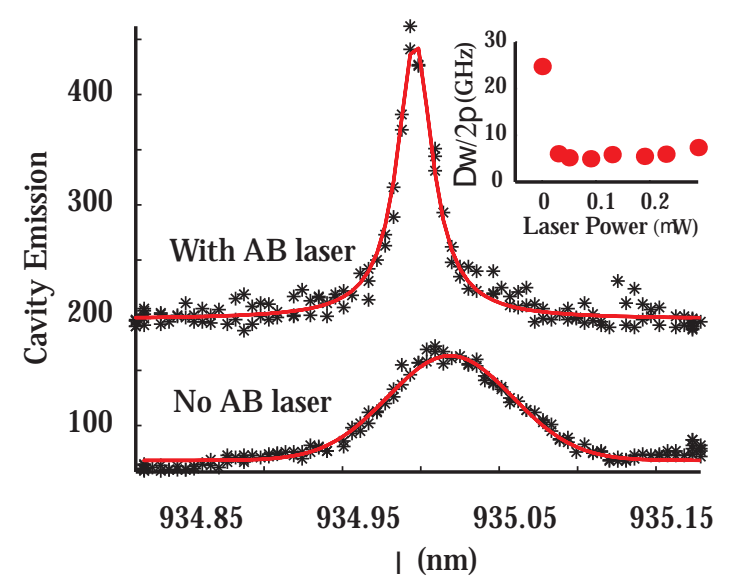

FIG. 4: (color online) Effect of the AB laser on the QD spectrum as measured though off-resonant cavity emission. In the absence of the AB laser, we observe a broad QD linewidth. However, in the presence of this laser, a significant linewidth narrowing is observed. The inset shows the QD linewidth measured as a function of the AB laser power.

citonic states are multiply charged states arising from the capture of multiple photo-generated carriers [26]. Figure $5 \mathrm{~b}$ shows the measured QD linewidth as a function of the excitation laser power both in the presence and absence of the $\mathrm{AB}$ laser. We find that the $\mathrm{QD}$ linewidth is nar- rower with $\mathrm{AB}$ laser on, at lower laser excitation power. In fact, the QD linewidth in that case follows ordinary power broadening (as shown by the solid line fit), with $\Delta \omega_{0} / 2 \pi=4.7 \mathrm{GHz}$, the same as the Lorentzian linewidth obtained from the fit to the linewidth data with spectral diffusion.

In summary, we have presented experimental studies showing the effect of the photo-generated carriers on spectral diffusion in the QD-cavity QED system. We first showed that the transmission spectrum of the strongly coupled QD-cavity system can be modified with a very low power of the additional above-band laser. We also show the significant effect of spectral diffusion in resonant QD spectroscopy, when the QD is embedded in a photonic crystal cavity. This spectral diffusion can be mitigated by photo-generated carriers that serve to fill the trap states provided by the etched surfaces of the PC. This significant reduction of the QD linewidth by $\mathrm{AB}$ excitation is an attractive prospect for potential quantum information applications employing QDs in PC structures. For those applications benefiting from narrow QD linewidths, the results presented here demonstrate a relatively simple means of improving the optical quality of QDs and thus system performance.

The authors acknowledge financial support provided by the NSF, ARO, and ONR. E.K. acknowledges support from the IC Postdoctoral Research Fellowship. The authors acknowledge Dr. Pierre Petroff and Dr. Hyochul Kim for providing the QD sample.
[1] A. Faraon, A. Majumdar, D. Englund, E. Kim, M. Bajcsy, and J. Vuckovic, New J. Physics 13, 055025 (2011).

[2] D. Englund, A. Faraon, I. Fushman, N. Stoltz, P. Petroff, and J. Vuckovic, Nature 450, 857 (2007).

[3] A. Imamoglu, D. Awschalom, G. Burkard, D. P. DiVincenzo, D. Loss, M. Sherwin, and A. Small, Phys. Rev. Lett. 83, 4204 (1999).

[4] D. Englund, A. Majumdar, A. Faraon, M. Toishi, N. Stoltz, P. Petroff, and J. Vučković, Phys. Rev. Lett. 104, 073904 (2010).

[5] S. Ates, S. M. Ulrich, A. Ulhaq, S. Reitzenstein, A. Lffler, S. Hfling, A. Forchel, and P. Michler, Nature Photonics 3, 724 (2009).

[6] C. Roy and S. Hughes, Phys. Rev. Lett. 106, 247403 (2011).

[7] A. Majumdar, A. Papageorge, E. D. Kim, M. Bajcsy, H. Kim, P. Petroff, and J. Vuckovic, arXiv:1103.2716 (2011).

[8] A. Berthelot, I. Favero, G. Cassabois, C. Voisin, C. Delalande, P. Roussignol, R. Ferreira, and J. M. Grard, Nature Physics 2, 759 (2006).

[9] H. D. Robinson and B. B. Goldberg, Phys. Rev. B 61, R5086 (2000).

[10] J. Seufert, R. Weigand, G. Bacher, T. Kummell, A. Forchel, K. Leonardi, and D. Hommel, Applied Physics Letters 76, 1872 (2000).

[11] V. Türck, S. Rodt, O. Stier, R. Heitz, R. Engelhardt, U. W. Pohl, D. Bimberg, and R. Steingrüber, Phys. Rev.
B 61, 9944 (2000).

[12] H. Kamada and T. Kutsuwa, Phys. Rev. B 78, 155324 (2008).

[13] G. Sallen, A. Tribu, T. Aichele, R. André, L. Besombes, C. Bougerol, M. Richard, S. Tatarenko, K. Kheng, and J.-P. Poizat, Phys. Rev. B 84, 041405 (2011).

[14] G. Sallen, A. Tribu, T. Aichele, R. Andr, L. Besombes, C. Bougerol, M. Richard, S. Tatarenko, K. Kheng, and J.-P. Poizat, Nature Photonics 4, 696 (2010).

[15] R. Heitz, T. R. Ramachandran, A. Kalburge, Q. Xie, I. Mukhametzhanov, P. Chen, and A. Madhukar, Phys. Rev. Lett. 78, 4071 (1997).

[16] C. F. Wang, A. Badolato, I. Wilson-Rae, P. M. Petroff, E. Hu, J. Urayama, and A. Imamoglu, Applied Physics Letters 85 (2004).

[17] A. Majumdar, A. Faraon, E. D. Kim, D. Englund, H. Kim, P. Petroff, and J. Vučković, Phys. Rev. B 82, 045306 (2010).

[18] R. Kubo, A Stochastic Theory of Line Shape (John Wiley and Sons, Inc., 2007), pp. 101-127.

[19] E. D. Kim, A. Majumdar, H. Kim, P. Petroff, and J. Vuckovic, Applied Physics Letters 97, 053111 (pages 3) (2010).

[20] D. Englund, A. Faraon, A. Majumdar, N. Stoltz, P. Petroff, and J. Vuckovic, Optics Express 17, 18651 (2009).

[21] A. Faraon, A. Majumdar, H. Kim, P. Petroff, and J. Vučković, Phys. Rev. Lett. 104, 047402 (2010). 


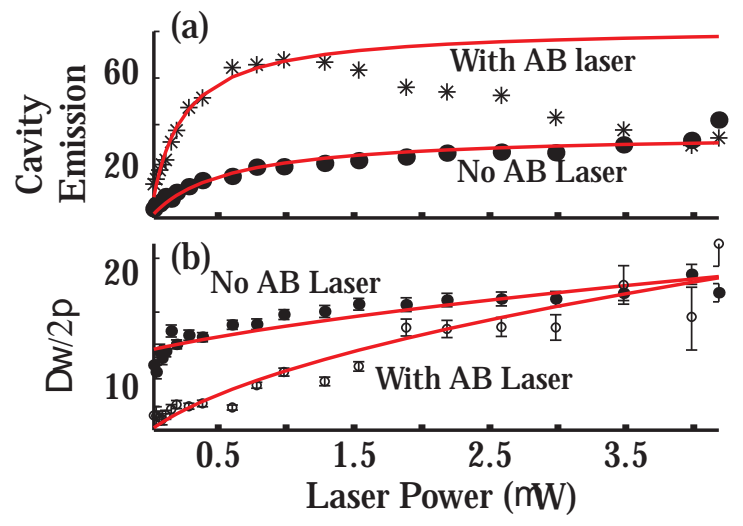

FIG. 5: (color online) (a) Off-resonant cavity emission as a function of the laser power driving the QD resonantly for two cases: with and without the additional, weak above band laser. (b) QD linewidth as a function of the resonant driving laser power. A much narrower linewidth is observed in the presence of the above-band laser at lower excitation powers. Also, the QD linewidth follows a simple power broadening with the excitation laser power when the above-band laser is present. In the absence of the above-band laser, we observe an additional power independent broadening, which can be explained by spectral diffusion of the QD.
[22] E. B. Flagg, A. Muller, J. W. Robertson, S. Founta, D. G. Deppe, M. Xiao, W. Ma, G. J. Salamo, and C. K. Shih, Nature Physics 5, 203 (2009).

[23] A. Majumdar, E. Kim, Y. Gong, M. Bajcsy, and J. Vuckovic, arXiv:1012.3125 (2011).

[24] A. J. Ramsay, A. V. Gopal, E. M. Gauger, A. Nazir, B. W. Lovett, A. M. Fox, and M. S. Skolnick, Phys. Rev. Lett. 104, 017402 (2010).

[25] J. Olivero and R. Longbothum, Journal of Quantitative Spectroscopy and Radiative Transfer 17, 233 (1977), ISSN 0022-4073.

[26] R. J. Warburton, C. Schflein, D. Haft, F. Bickel, A. Lorke, K. Karrai, J. M. Garcia, W. Schoenfeld, and P. M. Petroff, Nature 405, 916 (2000). 